\title{
First-principles study of two-dimensional van der Waals heterojunctions
}

\author{
Wei $\mathrm{Hu}^{1,2}$ and Jinlong Yang ${ }^{1,3, *}$ \\ ${ }^{1}$ Hefei National Laboratory for Physical Sciences at Microscale, \\ University of Science and Technology of China, Hefei, Anhui 230026, China \\ ${ }^{2}$ Computational Research Division, Lawrence Berkeley National Laboratory, Berkeley, CA 94720, USA \\ ${ }^{3}$ Synergetic Innovation Center of Quantum Information and Quantum Physics, \\ University of Science and Technology of China, Hefei, Anhui 230026, China
}

(Dated: May 5, 2015)

\begin{abstract}
Research on graphene and other two-dimensional (2D) materials, such as silicene, germanene, phosphorene, hexagonal boron nitride (h-BN), graphitic carbon nitride $\left(\mathrm{g}-\mathrm{C}_{3} \mathrm{~N}_{4}\right)$, graphitic zinc oxide $(\mathrm{g}-\mathrm{ZnO})$ and molybdenum disulphide $\left(\mathrm{MoS}_{2}\right)$, has recently received considerable interest owing to their outstanding properties and wide applications. Looking beyond this field, combining the electronic structures of 2D materials in ultrathin van der Waals heterojunctions has also emerged to widely study theoretically and experimentally to explore some new properties and potential applications beyond their single components. Here, this article reviews our recent theoretical studies on the structural, electronic, electrical and optical properties of 2D van der Waals heterojunctions using density functional theory calculations, including the Graphene/Silicene, Graphene/Phosphorene, Graphene/g-ZnO, Graphene/ $\mathrm{MoS}_{2}$ and $\mathrm{g}-\mathrm{C}_{3} \mathrm{~N}_{4} / \mathrm{MoS}_{2}$ heterojunctions. Our theoretical simulations, designs and calculations show that novel $2 \mathrm{D}$ van der Waals heterojunctions provide a promising future for electronic, electrochemical, photovoltaic, photoresponsive and memory devices in the experiments.
\end{abstract}

\section{INTRODUCTION}

Two-dimensional (2D) ultrathin materials, such as graphene [1-3], silicene [4-7], germanene [8-10], phosphorene [11-13], hexagonal boron nitride (h-BN) [14$16]$, graphitic carbon nitride $\left(\mathrm{g}-\mathrm{C}_{3} \mathrm{~N}_{4}\right)$ [17-19], graphitic zinc oxide $(\mathrm{g}-\mathrm{ZnO})$ [20-22] and molybdenum disulphide $\left(\mathrm{MoS}_{2}\right)$ [23-25], have received considerable interest recently owing to their outstanding properties and wide applications. There already have been many review articles [26-32] for the research on $2 \mathrm{D}$ materials over the past several years.

Graphene [2], a 2D sp²-hybridized carbon monolayer, is known to have remarkable electronic properties, such as a high carrier mobility, but the absence of a bandgap limits its applications of large-off current and high on-off ratio for graphene-based electronic devices. The same limitation also exists in silicene [4] and germanene [8], which have most similar remarkable electronic properties to graphene but with buckled honeycomb structures. On the other hand, most of graphene's derivatives, such as graphene oxide [33-35], graphane [36-38] and fluorographene [39-41] are all semiconductors but with lower carrier mobility, and have also been studied experimentally and theoretically.

Phosphorene, a new two dimensional (2D) elemental monolayer [11], has recently been experimentally isolated through mechanical exfoliation from bulk black phosphorus. Phosphorene exhibits some remarkable electronic properties superior to graphene. For example, phosphorene is a direct semiconductor with a high hole mobility [12], showing the drain current modulation up to $10^{5}$ in nanoelectronics [13]. Besides graphene, phosphorene is the only stable elemental 2D monolayer which can be mechanically exfoliated experimentally [12]. Most recently, arsenene and antimonene have also predicted theoretically [42]. Monolayer arsenene and antimonene are indirect wide-band-gap semiconductors, but become direct band-gap semiconductors under strain. Owing to these band-gap transitions, they can be used in nanoelectronic and optoelectronic devices.

2D h-BN monolayer [14], so-called "white graphene", is comprised of alternating boron and nitrogen atoms in a $\mathrm{sp}^{2}$-bonded 2D honeycomb arrangement and isolated from bulk BN. It has a wide bandgap used for its eccellent electrical insulations, high thermal conductivity and superior lubricant properties, and can be useful as an important complementary $2 \mathrm{D}$ dielectric substrate for graphene electronics.

$2 \mathrm{D}$ g- $\mathrm{C}_{3} \mathrm{~N}_{4}$ monolayer [17], which can be made by polymerization of cyanamide, dicyandiamide or melamine, is a semiconductor with a bandgap of about $2.7 \mathrm{eV}$, as a new polymeric photocatalyst for hydrogen production from water splitting under visible light, which has been demonstrated to be a be a potential candidate for solar cell absorber and photovoltaic materials.

$\mathrm{ZnO}$ nanofilms and nanoparticles have been widely used with graphene as composite materials for lightemitting diodes [43], photocatalysts [44], quantum dots [45] and field effect transistors [46]. Interestingly, it is predicted by first-principles calculations [20,21] and then confirmed experimentally [22] that $\mathrm{ZnO}(0001)$ film prefers a graphitic honeycomb structure when the layer number is reduced. $2 \mathrm{D} \mathrm{g}-\mathrm{ZnO}$ monolayer is also a semiconductor with a wide band gap of $3.57 \mathrm{eV}$, showing many interesting electronic and magnetic properties [4749]. 
$\mathrm{MoS}_{2}$ is an important kind of transition metal dichalcogenides (TMDCs), which has been widely studied experimentally and theoretically [23-25]. $\mathrm{MoS}_{2}$ has a direct band gap of about $2 \mathrm{eV}$, showing a high on-off current ratio with a high carrier mobility of around 200-500 $\mathrm{cm}^{2} /(\mathrm{Vs})$ in nanotransistors. Most of other TMDCs [29], such as $\mathrm{MoSe}_{2}, \mathrm{WS}_{2}$ and $\mathrm{WSe}_{2}$, but have attracted relatively little attention despite the fact that they have similar electronic structures to $\mathrm{MoS}_{2}$.

In parallel with the great efforts on $2 \mathrm{D}$ materials, another research field has recently emerged has been gaining strength over the past several years. It deals with ultrathin 2D van der Waals heterojunctions and devices made layer by layer from 2D materials, stacking different 2D monolayers on the top of each other. Strong covalent bonds provide in-plane stability of $2 \mathrm{D}$ monolayers, whereas relatively weak van der Waals forces are sufficient to keep the stack together. The possibility of making 2D van der Waals heterojunctions has been demonstrated experimentally for electronic, electrochemical, photovoltaic, photoresponsive and memory devices. Recently, there only have been several review articles [5053 ] involved for the research on 2D van der Waals heterojunctions.

Up to now, significant experimental and theoretical efforts have been made to characterize and explore new structures, properties and applications of 2D van der Waals heterojunctions, especially graphene based heterojunctions, such as graphene/hexagonal boron nitride (G/h-BN) [54-58], graphene/silicene (G/S) [59-61], graphene/phosphorene (G/P) [62-64], graphene/graphitic carbon nitride $\left(\mathrm{G} / \mathrm{g}-\mathrm{C}_{3} \mathrm{~N}_{4}\right) \quad[65-$ 67], graphene/graphitic zinc oxide ( $\mathrm{G} / \mathrm{g}-\mathrm{ZnO})$ [68-70], graphene/molybdenum disulphide $\left(\mathrm{G} / \mathrm{MoS}_{2}\right)$ [71-75], graphene/molybdenum disulfide $\left(\mathrm{G} / \mathrm{MoSe}_{2}\right)$ [76-78], silicene/hexagonal boron nitride (S/h-BN) [79-81], silicene/molybdenum disulphide $\left(\mathrm{S} / \mathrm{MoS}_{2}\right)$ [82-84], and TMDCs based heterojunctions [85-87]. These van der Waals heterojunctions show much more new properties far beyond their single components. Furthermore, most of them are ideal substrates for each other to preserve their intrinsic electronic properties due to weak van der Waals interactions at the interface.

Hybrid G/h-BN heterojunctions [54-58] are the most widely studied topic in $2 \mathrm{D}$ van der Waals heterojunctions during the past few years. Theoretically, Giovannetti et al. [54] proved that substrate-induced band gaps can be opened at the Dirac point of graphene on h-BN surfaces and the gap values are tunable as varying the the interfacial distance, which greatly improves the room temperature pinch-off characteristics of graphene-based field effect transistors. Experimentally, Dean et al. [55] first in 2010 introduced atomically thin 2D van der Waals heterojunctions in which graphene is on top of h-BN layers, and shown that h-BN can be used as an excellent complementary 2D dielectric substrate for graphene electronics.
In the present work, we review our recent theoretical studies on the structural, electronic, optical and electrical properties of $2 \mathrm{D}$ van der Waals heterojunctions with density functional theory calculations and shows that these 2D van der Waals heterojunctions provide a promising future for electronic, electrochemical, photovoltaic, photoresponsive and memory devices [50-53].

\section{THEORETICAL MODELS}

In order to simulate hybrid composite structures of $2 \mathrm{D}$ van der Waals heterojunctions made layer by layer, we need to choose two special supercells for each 2D material with different crystal systems and lattice constants, making them have a smaller lattice mismatch for each other. We have written a small program named LatticeMatch(See the supplementary material) to construct theoretical models for 2D van der Waals heterojunctions with a smaller lattice mismatch for different $2 \mathrm{D}$ materials. Its basic idea is based on the rotation matrix of extended orthogonal lattices for different 2D materials to search their minimum matching supercell models in $2 \mathrm{D}$ van der Waals heterojunctions.

\section{COMPUTATIONAL METHODS}

Our first-principles calculations are based on the density functional theory (DFT) implemented in the VASP package.[88] All the geometry structures are fully relaxed until energy and forces are converged to $10^{-5} \mathrm{eV}$ and 0.01 $\mathrm{eV} / \AA$, respectively. Dipole correction is employed to cancel the errors of electrostatic potential, atomic forces and total energy, caused by periodic boundary condition [89]. Very fine points in the surface Brillouin zone sampled with a regular mesh are used for calculating the tiny band gaps at the Dirac points of graphene and silicene in our recently studied $2 \mathrm{D}$ van der Waals heterojunctions.

Because conventional exchange-correlation functionals in the DFT calculations, such as the generalized gradient approximation of Perdew, Burke, and Ernzerhof (GGAPBE) [90], fail to adequately describe the long-range weak van der Waals interactions, different methods, such as semi-empirical long-range dispersion correction (DFTD) proposed by Grimme et al. [91-93] and non-empirical van der Waals density functional (vdW-DF) scheme proposed by Dion et al. [94-96], have been developed. Table I shows different methods implemented in VASP calculated equilibrium interfacial distance and binding energy per carbon atom for bilayer graphene $(a=b=2.47$ A) compared with experimental measurements [97, 98]. In our recent theoretical studies on $2 \mathrm{D}$ van der Waals heterojunctions, we choose the semi-empirical DFT-D2 method [92] due to its widely used and good description of long-range weak van der Waals interactions in molecu- 
TABLE I: Different methods implemented in VASP calculated equilibrium interfacial distance $D_{0}(\AA)$ and binding energy per carbon atom $E_{b}(\mathrm{meV})$ for bilayer graphene compared with experimental measurements.

\begin{tabular}{ccc}
\hline \hline Bilayer graphene & $D_{0}$ & $E_{b}$ \\
\hline GGA (PBE) & 3.90 & -0.04 \\
DFT-D2 (PBE) & 3.25 & -25.2 \\
vdW-DF (PBE) & 3.47 & -33.6 \\
vdW-DF (optB88) & 3.32 & -32.0 \\
Exp. [97, 98] & $3.35-26.0$ \\
\hline \hline
\end{tabular}

lar surface adsorption and layered structure systems [99109].

The charge carrier (hole and electron) concentration of doping graphene in 2D van der Waals heterojunctions can be estimated by the linear dispersion around the Dirac point of graphene[3]

$$
N_{h / e}=\frac{\left(\triangle E_{D}\right)^{2}}{\pi\left(\hbar \nu_{F}\right)^{2}}
$$

where $\triangle E_{D}$ is the shift of graphene's Dirac point $\left(E_{D}\right)$ relative to the Fermi level $\left(E_{F}\right)$, that is $\triangle E_{D}=E_{D}$ $E_{F}$.

To study the optical properties of $2 \mathrm{D}$ van der Waals heterojunctions, the frequency-dependent dielectric matrix is calculated [110]. The imaginary part of dielectric matrix is determined by a summation over states as

$$
\begin{aligned}
\varepsilon_{\alpha \beta}^{\prime \prime}= & \frac{4 \pi^{2} e^{2}}{\Omega} \lim _{q \rightarrow 0} \frac{1}{q^{2}} \sum_{c, v, \mathbf{k}} 2 w_{\mathbf{k}} \delta\left(\epsilon_{c \mathbf{k}}-\epsilon_{v \mathbf{k}}-\omega\right) \\
& \times\left\langle\mu_{c \mathbf{k}+\mathbf{e}_{\alpha} q} \mid \mu_{v \mathbf{k}}\right\rangle\left\langle\mu_{c \mathbf{k}+\mathbf{e}_{\beta} q} \mid \mu_{v \mathbf{k}}\right\rangle^{*}
\end{aligned}
$$

where, $\Omega$ is the volume of the primitive cell, $w_{\mathbf{k}}$ is the $\mathbf{k}$ point weight, $c$ and $v$ are the conduction and valence band states respectively, $\epsilon_{c \mathbf{k}}$ and $\mu_{c \mathbf{k}}$ are the eigenvalues and wavefunctions at the $\mathbf{k}$ point respectively, and $\mathbf{e}_{\alpha}$ are the unit vectors for the three Cartesian directions. In order to accurately calculate the optical properties of van der Waals heterojunctions, a large four times regular mesh for the surface Brillouin zone, a large number of empty conduction band states (two times more than the number of valence band) and frequency grid points are adopted in optical properties calculations compared with conventional electronic structure calculations.

\section{VAN DER WAALS HETEROJUNCTIONS}

We first check some common properties in our recently studied 2D van der Waals heterojunctions, including the Graphene/Silicene (G/S) [59], Graphene/Phosphorene (G/P) [62], Graphene/g-ZnO
TABLE II: DFT-D2 calculated equilibrium interfacial distance $D_{0}(\AA)$ and binding energy per carbon atom $E_{b}(m e V)$ in our studied $2 \mathrm{D}$ van der Waals heterojunctions

\begin{tabular}{ccc}
\hline \hline DFT-D2 & $D_{0}$ & $E_{b}$ \\
\hline Graphene/Silicene & 3.37 & -24.7 \\
Graphene/Phosphorene & 3.43 & -24.7 \\
Graphene/g-ZnO & 3.14 & -51.0 \\
Graphene/ $\mathrm{MoS}_{2}$ & 3.37 & -20.5 \\
$\mathrm{~g}_{-} \mathrm{C}_{3} \mathrm{~N}_{4} / \mathrm{MoS}_{2}$ & 2.97 & -22.4 \\
\hline \hline
\end{tabular}

(G/g-ZnO) [68], Graphene/ $\mathrm{MoS}_{2} \quad\left(\mathrm{G} / \mathrm{MoS}_{2}\right) \quad$ [75] and $\mathrm{g}-\mathrm{C}_{3} \mathrm{~N}_{4} / \mathrm{MoS}_{2}$ [87] heterojunctions as shown in Fig. 1, 2, 3, 4 and 5, respectively. We find that the weak van der Waals interlayer interactions in $2 \mathrm{D}$ van der Waals heterojunctions can induce new properties and phenomena, such as bandgap opening, charge transfer and new optical absorption.

Firstly, we find that weak van der Waals interactions domain at the interfaces of these $2 \mathrm{D}$ van der Waals heterojunctions. Table II shows our recently studied 2D van der Waals heterojunctions all have typical vdW equilibrium spacings with corresponding small binding energies, thus, they can be used as ideal substrates for each other with their intrinsic electronic structures undisturbed in $2 \mathrm{D}$ van der Waals heterojunctions. Furthermore, we confirm that the small lattice mismatch of about $2 \%$ for special supercells of 2D materials obtained by our LatticeMatch program has little effect on their electronic properties in these 2D van der Waals heterojunctions as shown their electronic band structures and density of states in Fig. 1b, 2b, 3b, 4b and 5b.

Secondly, small band gaps are opened at the Dirac points of graphene in graphene based 2D van der Waals heterojunctions due to weak van der Waals interactions at the interfaces, though its linear Dirac-like dispersion relation around the Fermi level is still preserved. Notice that induced graphene band gaps in $2 \mathrm{D}$ van der Waals heterojunctions are typically sensitive to other external conditions, such as interlayer separation [56], showing that the band gap values increase gradually with the interlayer separation decrease, thus tunable, with potential applications for graphene-based field effect transistors.

Thirdly, charge redistribution and transfer always occur at the interfaces in 2D van der Waals heterojunctions due to the interlayer coupling. In our recently studied 2D van der Waals heterojunctions, the G/S heterojunction belongs to the Metal/Metal type, the G/g$\mathrm{ZnO}, \mathrm{G} / \mathrm{P}$, and $\mathrm{G} / \mathrm{MoS}_{2}$ heterojunctions belong to the Metal/Semiconductor type, the $\mathrm{g}_{-} \mathrm{C}_{3} \mathrm{~N}_{4} / \mathrm{MoS}_{2}$ heterojunction belongs to the Semiconductor/Semiconductor type. For metal, they have different work functions (The energy difference between the vacuum level and the Fermi level). For semiconductors, they have different ioniza- 

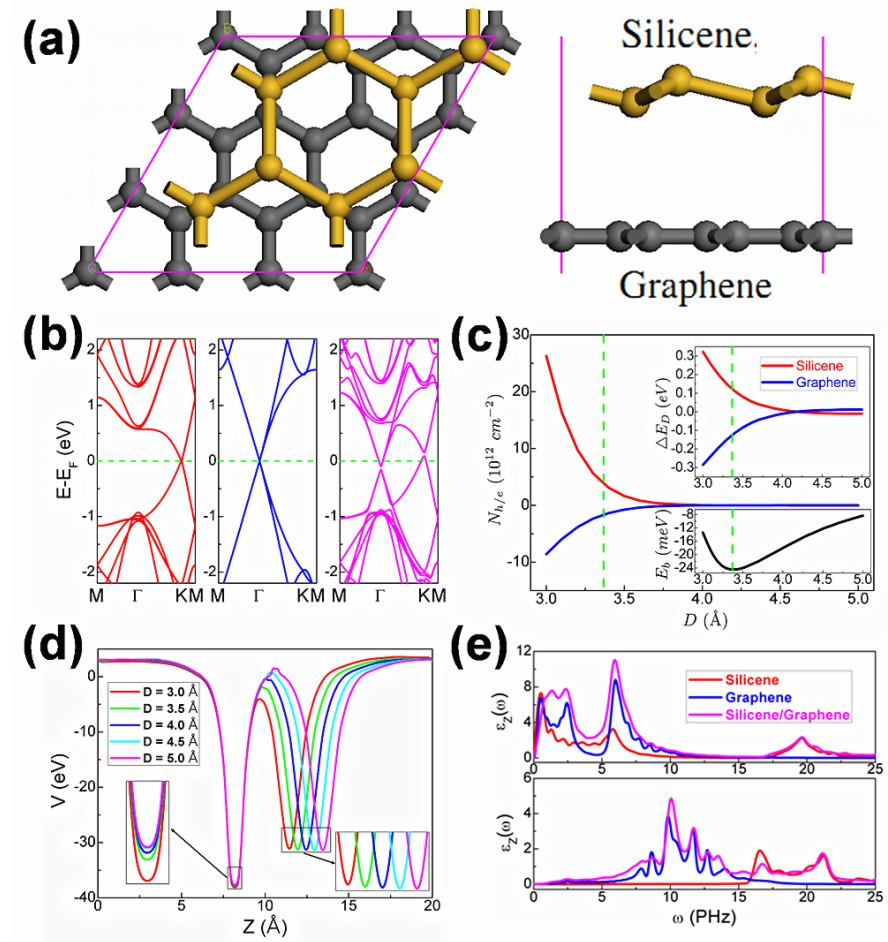

(e)

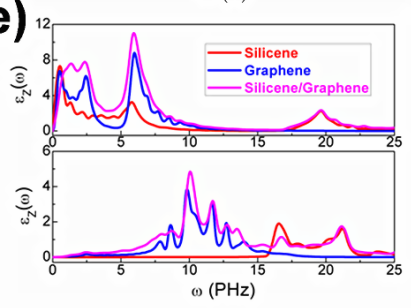

FIG. 1: (Color online) (a) Geometric structures of G/S heterojunction (top and side views). The gray and yellow balls denote carbon and silicon atoms, respectively. (b) Electronic band structures of silicene, graphene and G/S heterojunction. The Fermi level is set to zero and marked by green dotted lines. (c) The doping charge carrier concentrations $N_{h / e}\left(10^{12} \mathrm{~cm}^{-2}\right)$ of silicene and graphene of in $\mathrm{G} / \mathrm{S}$ heterojunction as a function of interfacial distance $D(\AA)$. The binding energies $E_{b}(m e V)$ per atom and the Dirac points $\triangle E_{D}(\mathrm{eV})$ of silicene and graphene shift relative to the Fermi level are shown in the inset. The equilibrium spacing is denoted by green dotted lines. (d) XY-averaged electrostatic potentials of G/S heterojunction at different interfacial distances $D(\AA)$ in the $\mathrm{Z}$ direction. Depths of potential wells of graphene and silicene are shown in the inset. (e) Imaginary part $\left(\epsilon^{\prime \prime}\right)$ of frequency $(E=\hbar \omega)$ dependent dielectric function (parallel and perpendicular) for pristine silicene and graphene monolayers as well as corresponding G/S heterojunction. (Reproduced from Ref. [59], with permission from American Institute of Physics.)

tions (The energy difference between the vacuum level and the conduction band minimum) and nucleophilics (The energy difference between the vacuum level and the valence band maximum) potentials. Based on the Schottky-Mott model [111], different work functions, ionizations and nucleophilics can induce charge transfer as well as well-separated electron-hole pairs at the interfaces in 2D van der Waals heterojunctions (Fig. 1c, 2e, $3 \mathrm{c}$ and 5a). Furtheremore, inhomogeneous surfaces also can results in charge redistribution and form intralayer electron-hole puddles at the interfaces in $2 \mathrm{D}$ van der Waals heterojunctions as shown in Fig. 3b. These new properties of charge redistribution and transfer at the interfaces can significantly enhance the conductivity and generate new catalytic activities in $2 \mathrm{D}$ van der Waals heterojunctions.

Fourthly, interlayer interaction [19] and charge transfer [67] in 2D van der Waals heterojunctions may induce new optical transitions, though pristine monolayers themselves display outstanding optical properties. We find that $2 \mathrm{D}$ van der Waals heterojunctions always ex- hibit wider absorption range and stronger optical absorption compared with their single monolayers as shown in Fig. 1e, 2e, 3d, 4d and 5c, because electrons can now be directly excited between different layers at the interfaces of heterojunctions. These new optical transitions in 2D van der Waals heterojunctions are expected to be with a great potential in photocatalytic and photovoltaic devices.

In the following of this review, we primarily focus on some special features of our recently studied 2D van der Waals heterojunctions that distinguish them from other previously studied 2D van der Waals heterojunctions.

\section{Graphene/Silicene}

Graphene and silicene are metallic but with different work functions, thus, both silicene and graphene are doped in hybrid G/S heterojunction, and their Dirac points respectively shift $0.12 \mathrm{eV}$ above and below the Fermi levels in hybrid G/S heterojunction, showing p- 
(a)
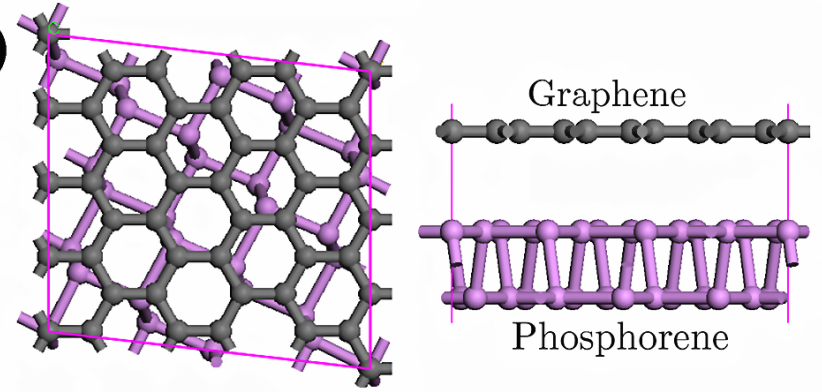

Phosphorene
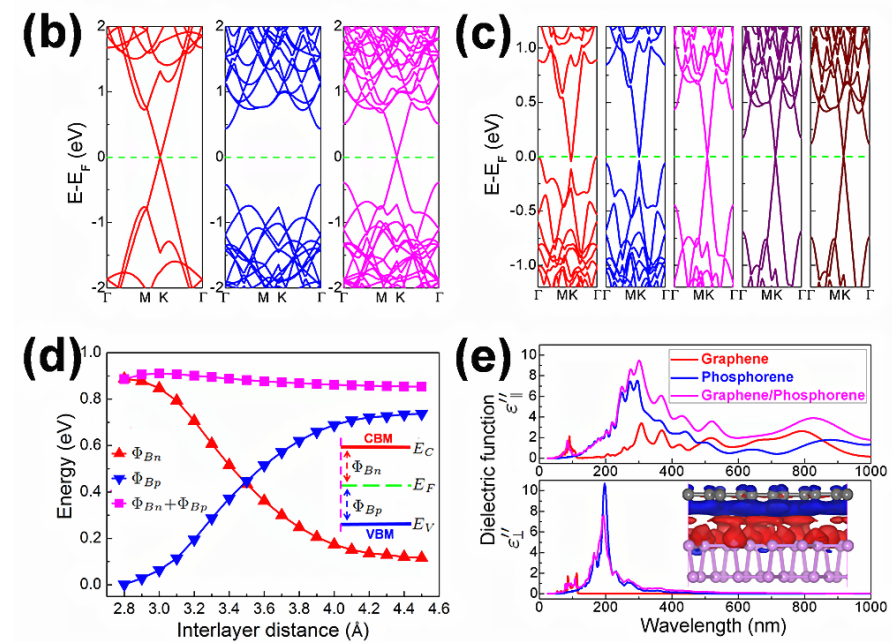

FIG. 2: (Color online) (a) Geometric structures of G/P heterojunction (top and side views). The gray and violet balls denote carbon and phosphorus atoms, respectively. (b) Electronic band structures of graphene, phosphorene and G/P heterojunction. The Fermi level is set to zero and marked by green dotted lines. (c) Electronic band structures of G/P heterojunction at different interfacial distances $D=2.8,3.0,3.5,4.0$ and $4.5 \AA$. (d) Schottky barriers $\Phi_{B n}, \Phi_{B p}$ and $\Phi_{B n}+\Phi_{B p}$ in G/P heterojunction as a function of interfacial distance. (e) Imaginary part of frequency dependent dielectric function (parallel and perpendicular) for pristine graphene and phosphorene monolayers as well as corresponding $\mathrm{G} / \mathrm{P}$ heterojunction. Differential charge density $\left(0.002 \mathrm{e} / \AA^{3}\right)$ of $\mathrm{G} / \mathrm{P}$ heterojunction is shown in the insert. The red and blue regions indicate electron increase and decrease, respectively. (Reproduced from Ref. [62], with permission from Royal Society of Chemistry.)

type and n-type doping of silicene and graphene, respectively. The calculated charge carrier concentrations are $N_{h}(\mathrm{~S})=3.8 \times 10^{12} \mathrm{~cm}^{-2}$ and $N_{e}(\mathrm{G})=1.6 \times 10^{12} \mathrm{~cm}^{-2}$ for silicene and graphene in hybrid $\mathrm{S} / \mathrm{G}$ nanocomposite, respectively. These values are more than 2 orders of magnitude larger than the intrinsic charge carrier concentration of graphene at room temperature $\left(n=\pi k_{B}^{2} T^{2} / 6 \hbar \nu_{F}^{2}=\right.$ $\left.6 \times 10^{10} \mathrm{~cm}^{-2}\right)$. Interestingly, the charge carrier concentrations of silicene and graphene in G/S heterojunction can be modulated with their interfacial spacing as shown in Fig. 1c. Furthermore, their charge carrier concentrations decrease gradually with their interlayer separation increase and a conversion of doping types of silicene and graphene happens when their interfacial distance artificially increases to above $4.2 \AA$. Therefore, selfdoping in $\mathrm{G} / \mathrm{S}$ heterojunction is effective and tunable with a great potential for new $\mathrm{p}-\mathrm{n}$ junctions.

We reveal the origin of tunable selfdoping in G/S heterojunction. When their interfacial distance artificially increases larger than $4.6 \AA, \triangle E_{D}$ is already unchanged for both silicene and graphene and converges to different values $\left(\triangle E_{D}(S)=-0.01 \mathrm{eV}\right.$ and $\triangle E_{D}(G)=0.01$ $e V)$. That is because silicene and graphene have different work functions, $W_{F}(S)=4.6 \mathrm{eV}$ and $W_{F}(G)=4.4 \mathrm{eV}$. Based on the Schottky-Mott model [111], electrons trend to transfer from graphene to silicene, resulting in weak n-type and p-type doping of silicene and graphene, respectively. This is an artificial effect induced by periodic boundary conditions in DFT calculations. But, when their interfacial distance decreases, weak overlap of electronic states between silicene and graphene is enhanced, which increase the charge transfer and electric double layer at the G/S interface, shifting up and down the energy levels of silicene and graphene respectively as shown in Fig. 1d. Note that there is a tunneling energy barrier for electrons at the $\mathrm{G} / \mathrm{S}$ interface, which is also reduced by their interface interactions. Moreover, silicene and graphene have different electronegativities (silicon 1.90 and carbon 2.55) and graphene has a more deeper potential well than silicene. Thus, when silicene and graphene 

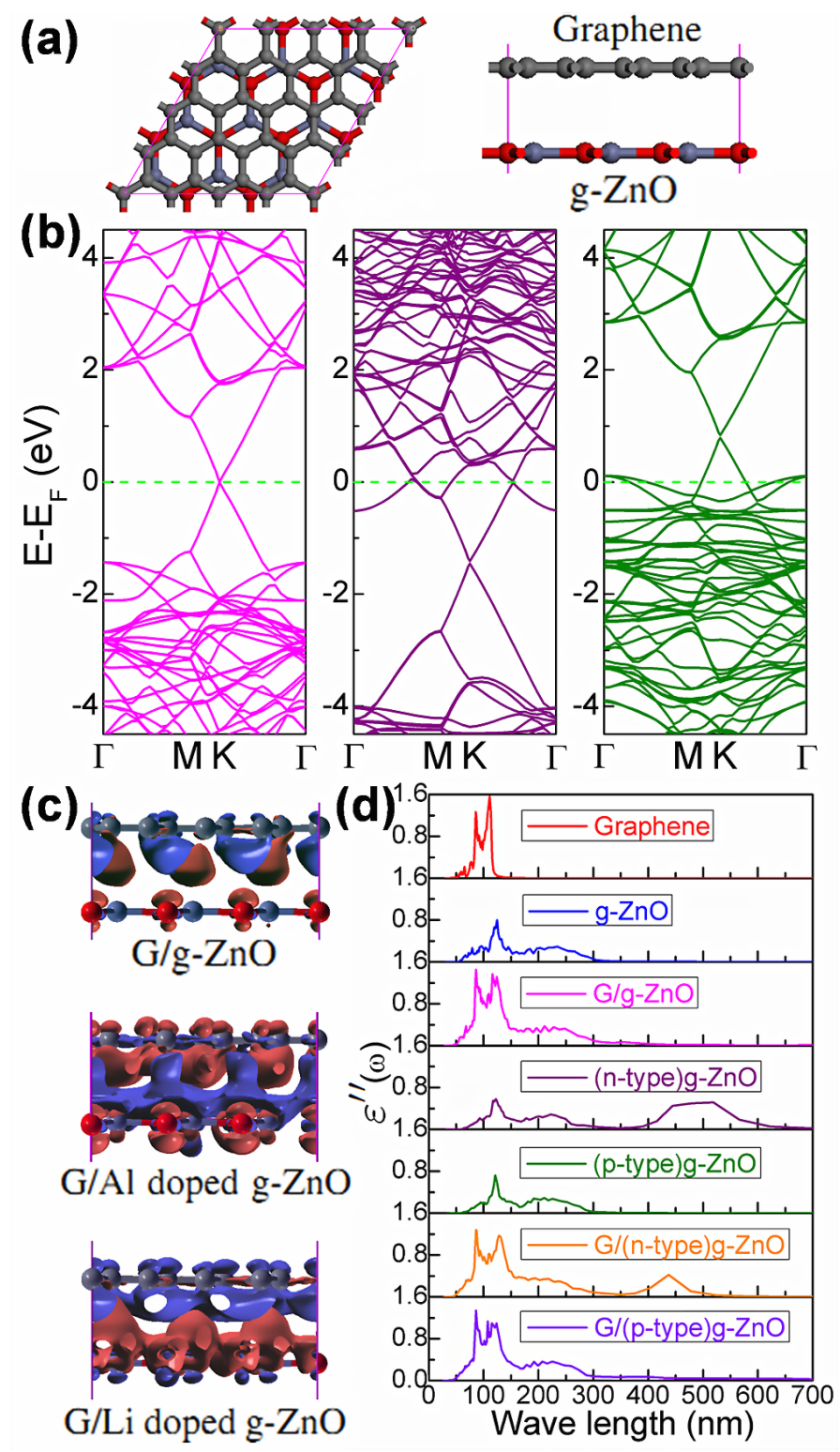

FIG. 3: (Color online) (a) Geometric structures structures of G/g-ZnO heterojunction (top and side views). The gray, red and blue balls denote carbon, oxygen and zinc atoms, respectively. (b) Electronic band structures of G/g-ZnO, G/Al doped $\mathrm{g}-\mathrm{ZnO}$, and $\mathrm{G} / \mathrm{Li}$ doped $\mathrm{g}-\mathrm{ZnO}$ heterojunctions. The Fermi level is set to zero and marked by red dotted lines. (c) Differential charge density $\left(0.002 e / \AA^{3}\right)$ for $\mathrm{G} / \mathrm{g}-\mathrm{ZnO}, \mathrm{G} / \mathrm{Al}$ doped $\mathrm{g}-\mathrm{ZnO}$, and $\mathrm{G} / \mathrm{Li}$ doped $\mathrm{g}-\mathrm{ZnO}$ heterojunctions. The red and blue regions indicate electron increase and decrease, respectively. (d) Imaginary part $\left(\epsilon^{\prime \prime}\right)$ of dielectric function (perpendicular) for graphene, g- $\mathrm{ZnO}$ monolayers and $\mathrm{G} / \mathrm{g}-\mathrm{ZnO}$ heterojunctions. (Reproduced from Ref. [68], with permission from American Institute of Physics.)

are close to each other (smaller than $4.2 \AA$ ), electrons are transferred from silicene to graphene, resulting in p-type and n-type doping of silicene and graphene, respectively. That is why a reversion of doping types of silicene and graphene happens when their interfacial distance is 4.2 $\AA$. Similarly, this feature of tunable doping types and charge carrier concentration of graphene on some metal surfaces [112] is also observed experimentally and theoretically.

\section{Graphene/Phosphorene}

Monolayer phosphorene is semiconducting with a direct band gap of $0.85 \mathrm{eV}$ (Fig. 2b) different form graphene. Therefore, a Schottky contact can be formed between metallic graphene and semiconducting phosphorene as shown in Fig. 2d. Based on the Schottky-Mott model [111] at the metal/semiconductor interface [113], a n-type Schottky barrier $\left(\Phi_{B n}\right)$ is defined as the energy difference between the Fermi level $\left(E_{F}\right)$ and the 

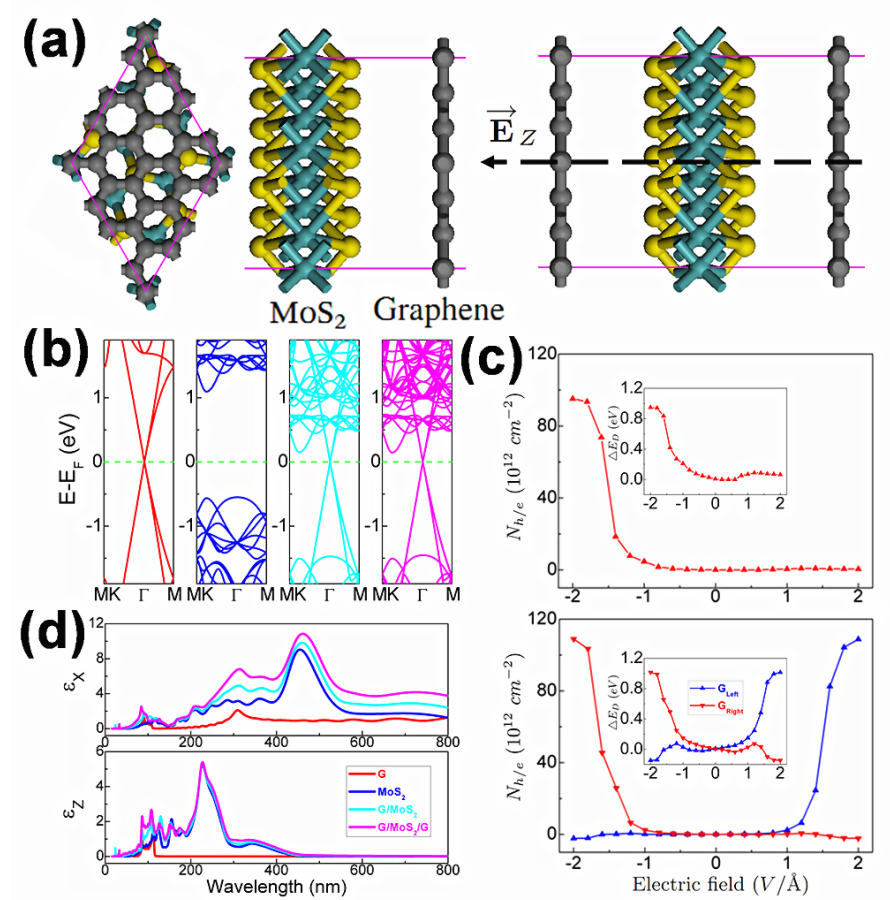

FIG. 4: (Color online) (a) Geometric structures of $\mathrm{G} / \mathrm{MoS}_{2}$ and $\mathrm{G} / \mathrm{MoS}_{2} / \mathrm{G}$ heterojunctions. The gray, yellow and blue balls denote carbon, sulfur and molybdenum atoms, respectively. (b) Electronic band structures of graphene and MoS $\mathrm{M}_{2}$ as well as corresponding $\mathrm{G} / \mathrm{MoS}_{2}$ and $\mathrm{G} / \mathrm{MoS}_{2} / \mathrm{G}$ heterojunctions. The Fermi level is set to zero and marked by green dotted lines. (c) The doping charge carrier concentrations $N_{h / e}\left(10^{12} \mathrm{~cm}^{-2}\right)$ of graphene in $\mathrm{G} / \mathrm{MoS}_{2}$ and $\mathrm{G} / \mathrm{MoS}_{2} / \mathrm{G}$ heterojunctions as a function of vertical electric field $E(V / \AA)$. The energy shift $\triangle E_{D}(e V)$ of graphene's Dirac point relative to the Fermi level is shown in the inset. (d) Imaginary part of dielectric function $\left(\varepsilon^{\prime \prime}\right)$ of pristine graphene $\mathrm{MoS}_{2}$ monolayers as well as corresponding $\mathrm{G} / \mathrm{MoS}_{2}$ and $\mathrm{G} / \mathrm{MoS}_{2} / \mathrm{G}$ heterojunctions. (Reproduced from Ref. [75])

conduction band minimum $\left(E_{C}\right)$, that is $\Phi_{B n}=E_{C}$ $E_{F}$. Similarly, a p-type Schottky barrier $\left(\Phi_{B p}\right)$ is defined as the energy difference between the Fermi level $\left(E_{F}\right)$ and valence band maximum $\left(E_{V}\right)$, that is $\Phi_{B p}=$ $E_{F}-E_{V}$. Notice that the sum of two types of Schottky barrier is approximately equal to the band gap value $\left(E_{G}\right)$ of semiconductor, that is $\Phi_{B n}+\Phi_{B p} \approx E_{G}$. As shown in Fig. 2c, the Dirac point of graphene moves from the conduction band to the valence band of phosphorene as the interfacial distance decreases from 4.5 to $2.8 \AA$, inducing a transition from n-type Schottky contact to ptype Schottky contact at the interface. The conversion of Schottky contact type in G/P heterojunction occurs when their interfacial distance increases to below $3.5 \AA$.

When the interfacial distance artificially increases larger than $4.5 \AA$, graphene's Dirac point is close to phosphorene's conduction band, forming a n-type Schottky contact with a small n-type Schottky barrier $\Phi_{B n}=0.12$ $\mathrm{eV}$ at the interface as shown in Fig. 2d. That is because graphene's work function $(4.3 \mathrm{eV})$ [59] is close to phosphorene's nucleophilic potential $(4.1 \mathrm{eV})$. As the interfacial distance decreases from 4.5 to $3.0 \AA$, the effects of charge transfer and chemical interactions (overlap of electronic states) between graphene and phosphorene are enhanced [113]. When the interfacial distance artificially decreases to $3.0 \AA$, graphene's Dirac point is close to phosphorene's valence band, forming a p-type Schottky contact with a negligible p-type Schottky barrier of $\Phi_{B p}$ $=0.06 \mathrm{eV}$ at the interface as shown in Fig. 2d. Furthermore, strong interlayer-interactions even can induce weak n-type doping in graphene when the interfacial distance artificially decreases to $2.8 \AA$ as shown in Fig. 2c. Therefore, $\mathrm{G} / \mathrm{P}$ heterojunction has tunable Schottky contacts and barriers as varying the the interfacial distance.

Notice that the Schottky contact in G/P heterojunction is very different to traditional metal-semiconductor Schottky ones in two important ways. One is that graphene is adsorbed physically on phosphorene in ultrathin van der Waals heterojunction without dangling bonds at the interface. Another is the Schottky contacts and barriers can be adjusted sensitively by varying the interfacial distance. Furthermore, atom doping in graphene and applying vertical electric fields can also be used to modify the work function of graphene and then to adjust the Schottky contacts and barriers in G/P heterojunction. Therefore, G/P heterojunction can be used for tunable Schottky diodes in nanoelectronics. 

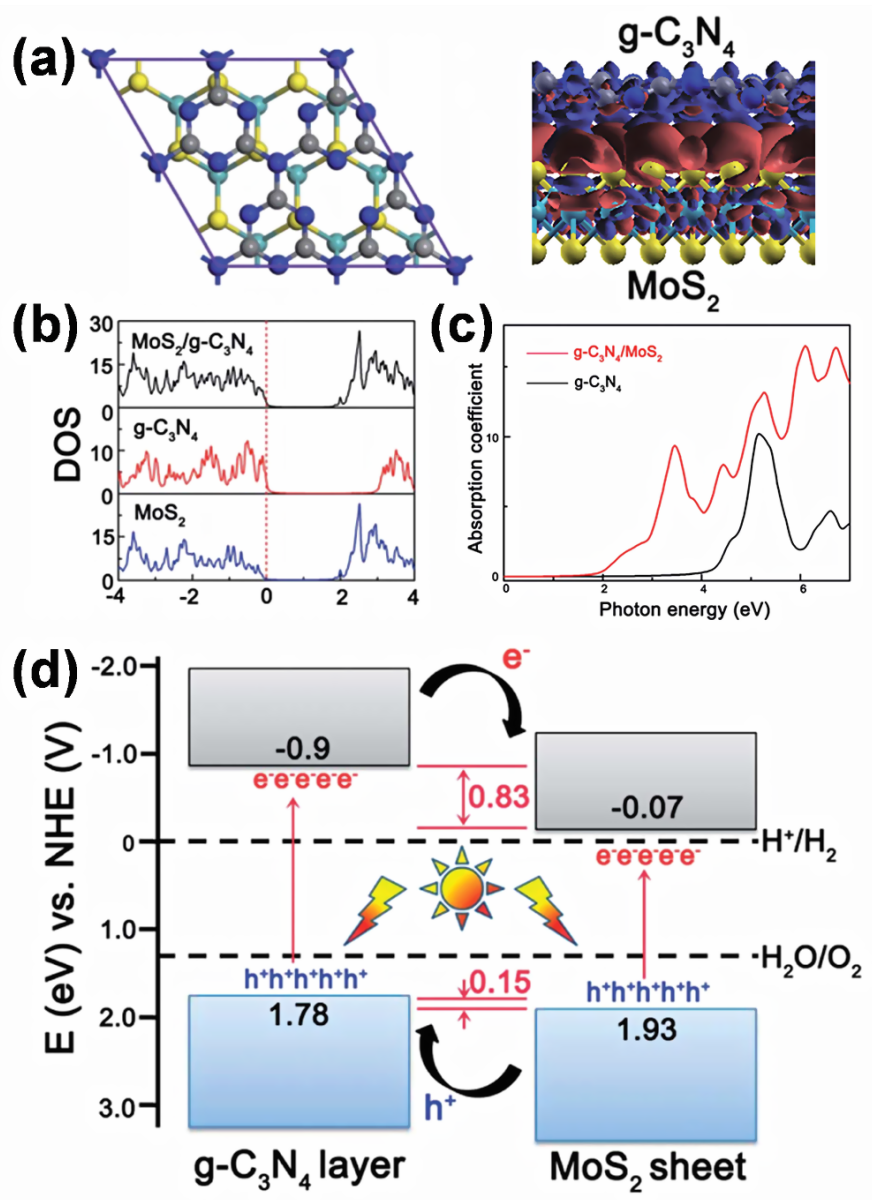

FIG. 5: (Color online) (a) Geometric structures and differential charge density of $\mathrm{g}-\mathrm{C}_{3} \mathrm{~N}_{4} / \mathrm{MoS}_{2}$ heterojunction. The gray, blue, yellow and blue balls denote carbon, nitrogen, sulfur and molybdenum atoms, respectively. The red and blue regions indicate electron increase and decrease, respectively. (b) Density of states of $\mathrm{g}-\mathrm{C}_{3} \mathrm{~N}_{4} / \mathrm{MoS}_{2}$ heterojunction, g-C $\mathrm{N}_{4}$ and $\mathrm{MoS}{ }_{2}$ monolayers. The Fermi level is set to zero and marked by green dotted lines. (c) Absorption coefficients of g- $\mathrm{C}_{3} \mathrm{~N}_{4}$ monolayer and $\mathrm{g}-\mathrm{C}_{3} \mathrm{~N}_{4} / \mathrm{MoS}_{2}$ heterojunction. (d) Schematic illustration of carrier transfer and separation in g- $\mathrm{C}_{3} \mathrm{~N}_{4} / \mathrm{MoS}_{2}$ heterojunction. (Reproduced from Ref. [87], with permission from American Chemical Society.)

\section{Graphene/g-ZnO}

Monolayer $\mathrm{g}-\mathrm{ZnO}$ is a wide-gap semiconductor as shown in Fig. 3b. When forming a G/g-ZnO heterojunction, its Fermi level remains in the induced gap, indicating that little charge transfer exits between graphene and undoped $\mathrm{g}-\mathrm{ZnO}$ monolayer. This is also supported by their differential charge density plotted in Fig. 3c. Notice that the inhomogeneous g- $\mathrm{ZnO}$ substrate induces charge redistribution in the graphene plane, forming intralayer electron-hole puddles, which may significantly enhance the electron conductivity and generate new photovoltaic and catalytic activities [67].

Different charge rearrangement occurs in G/doped g$\mathrm{ZnO}$ heterojunctions as shown in Fig. 3c. We find that $\mathrm{Al}$ and $\mathrm{Li}$ doped g-ZnO monolayers have a work function either $1.2 \mathrm{eV}$ smaller or the same amount larger than that of pristine graphene. Based on the Schottky-
Mott model[111], the Dirac point of the composite systems is moved to below and above the Fermi level (FIG $3 \mathrm{~b}$ and $3 \mathrm{c}$ ), respectively. As shown in Fig. 3c, electronhole pairs are well separated at the interfaces of $\mathrm{G} /$ doped g-ZnO heterojunctions. The calculated charge carrier density is $N_{e}(\mathrm{G} / \mathrm{n}$-type $\mathrm{g}-\mathrm{ZnO})=3.5 \times 10^{13} \mathrm{~cm}^{-2}$ and $N_{h}(\mathrm{G} / \mathrm{p}$-type $\mathrm{g}-\mathrm{ZnO})=1.1 \times 10^{13} \mathrm{~cm}^{-2}$. These values are more than 3 orders of magnitude larger than the intrinsic charge carrier concentration of graphene at room temperature $\left(n=\pi k_{B}^{2} T^{2} / 6 \hbar \nu_{F}^{2}=6 \times 10^{10} \mathrm{~cm}^{-2}\right)$. Compared to the charge carrier density of isolated $\mathrm{Al}$ and $\mathrm{Li}$ doped g-ZnO monolayers $\left(n=m \triangle E / \pi \hbar^{2}\right.$, where $\triangle E$ is the difference between the Fermi level and conduction band minimum or valence band maximum), the charge transfer efficiency is about $45 \%$ and $20 \%$, respectively. Therefore, the charge transfer at the G/doped g-ZnO interfaces can induce effective electron and hole doping in graphene for graphene-based Schottky diodes and p-n junctions. 


\section{Graphene/MoS 2}

High-performance field-effect tunneling transistors have been achieved experimentally [73, 74] in hybrid graphene and $\mathrm{MoS}_{2}$ heterojunctions. Thus, the electronic properties of hybrid $\mathrm{G} / \mathrm{MoS}_{2}$ and $\mathrm{G} / \mathrm{MoS}_{2} / \mathrm{G}$ heterojunctions affected by applying vertical electric fields are very desirable as shown in Fig. 4c. Interestingly, negative vertical electric fields can induce p-type doping of graphene in hybrid $\mathrm{G} / \mathrm{MoS}_{2}$ heterojunctions. But, positive electric fields almost have on effect on the electronic properties of hybrid $\mathrm{G} / \mathrm{MoS}_{2}$ heterojunctions. This is because electrons can easily from the Dirac point of graphene to the conduction band of $\mathrm{MoS}_{2}$ but difficulty from the valence band of $\mathrm{MoS}_{2}$ to the Dirac point of graphene due to the work function $(4.3 \mathrm{eV})$ [68] of graphene close to the electronic affinity $(4.2 \mathrm{eV})$ of monolayer $\mathrm{MoS}_{2}$.

Interestingly, vertical electric fields can generate strong p-type but weak n-type doping of graphene at both negative and positive electric fields due to the symmetry in hybrid $\mathrm{G} / \mathrm{MoS}_{2} / \mathrm{G}$ heterojunctions. The calculated charge carrier concentrations in hybrid $\mathrm{G} / \mathrm{MoS}_{2}$ and $\mathrm{G} / \mathrm{MoS}_{2} / \mathrm{G}$ heterojunctions are shown in Fig. 4c. These values are more than 3 orders of magnitude larger than the intrinsic charge carrier concentration of graphene at room temperature $\left(n=\pi k_{B}^{2} T^{2} / 6 \hbar \nu_{F}^{2}=6 \times 10^{10} \mathrm{~cm}^{-2}\right)$. Furthermore, the doping charge carrier concentrations of graphene in hybrid heterojunctions are increased with the vertical electric fields. Notice that the electron-hole pairs are well separated in hybrid $\mathrm{G} / \mathrm{MoS}_{2} / \mathrm{G}$ sandwiched heterojunctions with more excellent applications[73, 74] compared with hybrid $\mathrm{G} / \mathrm{MoS}_{2}$ heterojunctions. Therefore, the field-effect in hybrid graphene and $\mathrm{MoS}_{2}$ heterojunctions is effective and tunable for high-performance FETs and $\mathrm{p}-\mathrm{n}$ junctions.

$$
\text { g- } \mathrm{C}_{3} \mathrm{~N}_{4} / \mathrm{MoS}_{2}
$$

Monolayer $\mathrm{g}-\mathrm{C}_{3} \mathrm{~N}_{4}$ and $\mathrm{MoS}_{2}$ are all semiconductors with direct band gaps of 2.7 and $2.0 \mathrm{eV}$, respectively. g$\mathrm{C}_{3} \mathrm{~N}_{4}$ can be used to achieve both half reactions of water splitting under visible light, meaning that its bandgap cover both the water reduction and water oxidation potentials. However, the photocatalytic $\mathrm{H}_{2}$ production activity of pristine $\mathrm{g}-\mathrm{C}_{3} \mathrm{~N}_{4}$ remains poor, strongly depending on the surface co-catalysts. On the other hand, $\mathrm{MoS}_{2}$ has been extensively investigated as a promising electrocatalyst for $\mathrm{H}_{2}$ evolution due to its high abundance and low cost. According to some experimental studies, $\mathrm{MoS}_{2}$ can serve as a suitable co-catalysts to composite with g- $\mathrm{C}_{3} \mathrm{~N}_{4}$ to show enhanced photocatalytic performance.

Our calculated band alignment between the $\mathrm{g}-\mathrm{C}_{3} \mathrm{~N}_{4}$ and $\mathrm{MoS}_{2}$ monolayers reveals that the conduction band minimum and valence band maximum of the $\mathrm{g}-\mathrm{C}_{3} \mathrm{~N}_{4}$ monolayer are higher by about $0.83 \mathrm{eV}$ and $0.15 \mathrm{eV}$ respectively than those of the $\mathrm{MoS}_{2}$ monolayer. This predicted type-II band alignment ensures the photogenerated electrons easily migrate from the $\mathrm{g}-\mathrm{C}_{3} \mathrm{~N}_{4}$ monolayer to the $\mathrm{MoS}_{2}$ monolayer, and leads to the high hydrogenevolution reaction activity. The charge transfer between g- $\mathrm{C}_{3} \mathrm{~N}_{4}$ and $\mathrm{MoS}_{2}$ results in a polarized field within the interface region, which will benefit the separation of photogenerated carriers. The calculated optical absorption curves verify that this proposed layered nanocomposite is a good light-harvesting semiconductor. These findings indicate that $\mathrm{MoS}_{2}$ is a promising candidate as a non-noble metal co-catalyst for $\mathrm{g}-\mathrm{C}_{3} \mathrm{~N}_{4}$ photocatalysts, and also provide useful information for understanding the observed enhanced photocatalytic mechanism in experiments.

\section{SUMMARY AND CONCLUSIONS}

In the present work, we review our recent theoretical studies on the structural, electronic, optical and electrical properties of $2 \mathrm{D}$ van der Waals heterojunctions via density functional theory calculations, including the Graphene/Silicene, Graphene/Phosphorene, Graphene/g-ZnO, Graphene/ $\mathrm{MoS}_{2}$ and $\mathrm{g}-\mathrm{C}_{3} \mathrm{~N}_{4} / \mathrm{MoS}_{2}$ heterojunctions. We find that the weak interlayer van der Waals interactions in these 2D heterojunctions can induce new properties and phenomena, such as bandgap opening, charge transfer and new optical absorption. Furthermore, our recently studied 2D van der Waals heterojunctions have some special features which distinguish them from other previously studied 2D van der Waals heterojunctions. With excellent structural, electronic, electrical and optical properties properties combined, 2D van der Waals heterojunctions are expected to be with great applications in efficient electronic, electrochemical, photovoltaic, photoresponsive and memory devices.

\section{ACKNOWLEDGEMENTS}

This work is partially supported by the National Key Basic Research Program (2011CB921404), by NSFC (11404109, 21121003, 91021004, 21233007, 21222304), by CAS (XDB01020300). This work is also partially supported by the Scientific Discovery through Advanced Computing (SciDAC) program funded by U.S. Department of Energy, Office of Science, Advanced Scientific Computing Research and Basic Energy Sciences (W. H.). We thank the National Energy Research Scientific Computing (NERSC) center, and the USTCSCC, SC-CAS, Tianjin, and Shanghai Supercomputer Centers for the computational resources. 


\section{APPENDIX A. SUPPLEMENTARY MATERIAL}

Supplementary material associated with this review article can be found, in the online version, at http://dx.doi.org/10.XXXX/XX.XX.

\section{REFERENCES}

* Corresponding author. E-mail: jlyang@ustc.edu.cn

[1] K.S. Novoselov, A.K. Geim, S.V. Morozov, D. Jiang, Y. Zhang, S.V. Dubonos, I.V. Grigorieva, A.A. Firsov, Scinece 306 (2004) 666.

[2] A.K. Geim, K.S. Novoselov, Nature Mater. 6 (2007) 183.

[3] A.H.C. Neto, F. Guinea, N.M.R. Peres, K.S. Novoselov, A.K. Geim, Rev. Mod. Phys. 81 (2009) 109.

[4] S. Cahangirov, M. Topsakal, E. Aktürk, H. Şahin, S. Ciraci, Phys. Rev. Lett. 102 (2009) 236804.

[5] P. Vogt, P. D. Padova, C. Quaresima, J. Avila, E. Frantzeskakis, M.C. Asensio, A. Resta, B. Ealet, G.L. Lay, Phys. Rev. Lett. 108 (2012) 155501.

[6] A. Kara, H. Enriquez, A.P. Seitsonend, L.C.L.Y. Voone, S. Vizzini, B. Aufrayg, H. Oughaddoub, Surf. Sci. Rep. 67 (2012) 1.

[7] H. Oughaddou, H. Enriquez, M.R. Tchalala, H. Yildirim, A.J. Mayne, A. Bendounan, G. Dujardin, M.A. Ali, A. Kara, Prog. Surf. Sci. 90 (2015) 46.

[8] E. Bianco, S. Butler, S. Jiang, O.D. Restrepo, W. Windl, J.E. Goldberger, ACS Nano 7 (2013) 4414.

[9] L. Li, S.-Z. Lu, J. Pan, Z. Qin, Y.-Q. Wang, Y. Wang, C.-Y. Cao, S. Du, H.-J. Gao, Adv. Mater. 26 (2014) 4820.

[10] M.E. Dávila, L. Xian, S. Cahangirov, A. Rubio, G.L. Lay, New J. Phys. 16 (2014) 095002.

[11] L. Li, Y. Yu, G. Ye, Q. Ge, X. Ou, H. Wu, D. Feng, X. Chen, Y. Zhang, Nature Nanotech. 9 (2014) 372.

[12] H. Liu, A.T. Neal, Z. Zhu, Z. Luo, X. Xu, D. Tománek, P.D. Ye, ACS Nano 8 (2014) 4033.

[13] J. Qiao, X. Kong, Z. Hu, F. Yang, W. Ji, Nature Commun. 5 (2014) 4475.

[14] K. Watanabe, T. Taniguchi, H. Kanda, Nature Mater. 3 (2004) 404.

[15] L. Song, L. Ci, H. Lu, P.B. Sorokin, C. Jin, J. Ni, A.G. Kvashnin, D.G. Kvashnin, J. Lou, B.I. Yakobson, P.M. Ajayan, Nano Lett. 10 (2010) 3209.

[16] Y. Shi, C. Hamsen, X. Jia, K.K. Kim, A. Reina, M. Hofmann, A.L. Hsu, K. Zhang, H. Li, Z.-Y. Juang, M.S. Dresselhaus, L.-J. Li, J, Kong, Nano Lett. 10 (2010) 4134.

[17] X. Wang, K. Maeda, A. Thomas, K. Takanabe, G. Xin, J.M. Carlsson, K. Domen, M. Antonietti, Nature Mater. 8 (2009) 76.

[18] X. Wang, K. Maeda, X. Chen, K. Takanabe, K. Domen, Y. Hou, X. Fu, M. Antonietti, J. Am. Chem. Soc. 131 (2009) 1680.

[19] F. Wu, Y. Liu, G. Yu, D. Shen, Y. Wang, E. Kan, J. Phys. Chem. Lett. 3 (2012) 3330.
[20] F. Claeyssens, C.L. Freeman, N.L. Allan, Y. Sun, M.N.R. Ashfold, J.H. Harding, J. Mater. Chem. 15 (2005) 139.

[21] C.L. Freeman, F. Claeyssens, N.L. Allan, Phys. Rev. Lett. 96 (2006) 066102.

[22] C. Tusche, H.L. Meyerheim, and J. Kirschner, Phys. Rev. Lett. 99 (2007) 026102.

[23] K.F. Mak, C. Lee, J. Hone, J. Shan, T.F. Heinz, Phys. Rev. Lett. 105 (1020) 136805.

[24] B. Radisavljevic, A. Radenovic, J. Brivio, V. Giacometti, A. Kis, Nature Nanotech. 6 (2011) 147.

[25] Z. Yin, H. Li, H. Li, L. Jiang, Y. Shi, Y. Sun, G. Lu, Q. Zhang, X. Chen, H. Zhang, ACS Nano 6 (2012) 74.

[26] K.S. Novoselov, D. Jiang, F. Schedin, T.J. Booth, V.V. Khotkevich, S.V. Morozov, A.K. Geim, Proc. Natl. Acad. Sci. USA 102 (2005) 10451.

[27] R. Mas-Ballesté, C. Gómez-Navarro, J. Gómez-Herrero, F. Zamora, Nanoscale 3 (2011) 20.

[28] M. Osada, T. Sasaki, Adv. Mater. 24 (2012) 210.

[29] Q.H. Wang, K. Kalantar-Zadeh, A. Kis, J.N. Coleman, M.S. Strano, Nature Nanotechnol. 7 (2012) 699.

[30] M. Xu, T. Liang, M. Shi, H. Chen, Chem. Rev. 113 (2013) 3766.

[31] S.Z. Butler, S.M. Hollen, L. Cao, Y. Cui, J.A. Gupta, H.R. Gutiérrez, T.F. Heinz, S.S. Hong, J. Huang, A.F. Ismach, E. Johnston-Halperin, M. Kuno, V.V. Plashnitsa, R.D. Robinson, R.S. Ruoff, S. Salahuddin, J. Shan, L. Shi, M.G. Spencer, M. Terrones, W. Windl, J.E. Goldberger, ACS Nano 7 (2013) 2898.

[32] S. Balendhran, S. Walia, H. Nili, S. Sriram, M. Bhaskaran, small 11 (2014) 640.

[33] D.A. Dikin, S. Stankovich, E.J. Zimney, R.D. Piner, G.H.B. Dommett, G. Evmenenko, S.T. Nguyen, R.S. Ruoff, Nature 448 (2007) 457.

[34] Y. Zhu, S. Murali, W. Cai, X. Li, J.W. Suk, J.R. Potts, R.S. Ruoff, Adv. Mater. 22 (2010) 3906.

[35] D.R. Dreyer, S. Park, C.W. Bielawski, R.S. Ruoff, Chem. Soc. Rev. 39 (2010) 228.

[36] J.O. Sofo, A.S. Chaudhari, G.D. Barber, Phys. Rev. B 75 (2007) 153401.

[37] M.Z.S Flores, P.A.S Autreto, S.B. Legoas, D.S. Galvao, Nanotechnology 20 (2009) 465704.

[38] D.C. Elias, R.R. Nair, T.M.G. Mohiuddin, S.V. Morozov, P. Blake, M.P. Halsall, A.C. Ferrari, D.W. Boukhvalov, M.I. Katsnelson, A.K. Geim, K.S. Novoselov, Science 323 (2009) 610.

[39] R.R. Nair, W. Ren, R. Jalil, I. Riaz, V.G. Kravets, L. Britnell, P. Blake, F. Schedin, A.S. Mayorov, S. Yuan, M.I. Katsnelson, H.-M. Cheng, W. Strupinski, L.G. Bulusheva, A.V. Okotrub, I.V. Grigorieva, A.N. Grigorenko, K.S. Novoselov, A.K. Geim, small 6 (2010) 2877.

[40] D.K. Samarakoon, Z. Chen, C. Nicolas, X.-Q. Wang, small 7 (2011) 965.

[41] K.-J. Jeon, Z. Lee, E. Pollak, L. Moreschini, A. Bostwick, C.-M. Park, R. Mendelsberg, V. Radmilovic, R. Kostecki, T.J. Richardson, E. Rotenberg, ACS Nano 5 (2011) 1042.

[42] S. Zhang, Z. Yan, Y. Li, Z. Chen, H. Zeng, Angew. Chem. 54 (2015) 3112.

[43] K. Chung, C.H. Lee, G.C. Yi, Science 330 (2010) 655.

[44] O. Akhavan, ACS Nano 4 (2010) 4174.

[45] D.I. Son, B.W. Kwon, D.H. Park, W.S. Seo, Y. Yi, B. Angadi, C.-L. Lee, W.K. Choi, Nature Nanotech. 
7 (2012) 465.

[46] Y.Y. Hui, G. Tai, Z. Sun, Z. Xu, N. Wang, F. Yan, S.P. Lau, Nanoscale 4 (2012) 3118.

[47] E.J. Kan, H.J. Xiang, F. Wu, C. Tian, C. Lee, J.L. Yang, M.-H. Whangbo, Appl. Phys. Lett. 97 (2010) 122503.

[48] T.M. Schmidt, R.H. Miwa, A. Fazzio, Phys. Rev. B 81 (2010) 195413.

[49] H. Guo, Y. Zhao, N. Lu, E. Kan, X.C. Zeng, X. Wu, J. Yang, J. Phys. Chem. C 116 (2012) 11336.

[50] C. Dean, A.F. Young, L. Wang, I. Meric, G.-H. Lee, K. Watanabe, T. Taniguchi, K. Shepard, P. Kim, J. Hone, Solid State Commun. 152 (2012) 1275.

[51] A.K. Geim, I.V. Grigorieva, Nature 499 (2013) 419.

[52] H. Wang, F. Liu, W. Fu, Z. Fang, W. Zhou, Z. Liu, Nanoscale 6 (2014) 12250.

[53] T. Niu, A. Li, Prog. Surf. Sci. 90 (2015) 21.

[54] G. Giovannetti, P.A. Khomyakov, G. Brocks, P.J. Kelly, J. van den Brink, Phys. Rev. B 76 (2007) 073103.

[55] C.R. Dean, A.F. Young, I. Meric, C. Lee, L. Wang, S. Sorgenfrei, K. Watanabe, T. Taniguchi, P. Kim, K.L. Shepard, J. Hone, Nature Nanotech. 5 (2010) 722.

[56] Y. Fan, M. Zhao, Z. Wang, X. Zhang, H. Zhang, Appl. Phys. Lett. 98 (2011) 083103.

[57] J. Xue, J. Sanchez-Yamagishi, D. Bulmash, P. Jacquod, A. Deshpande, K. Watanabe, T. Taniguchi, P. JarilloHerrero, B. LeRoy, Nature Mater. 10 (2011) 282.

[58] K.H. Lee, H.-J. Shin, J. Lee, I. Lee, G.-H. Kim, J.-Y. Choi, S.-W. Kim, Nano Lett. 12 (2012) 714.

[59] W. Hu, Z. Li, J. Yang, J. Chem. Phys. 139 (2013) 154704.

[60] Y. Cai, C.-P. Chuu, C.M. Wei, M.Y. Chou, Phys. Rev. B 88 (2013) 245408.

[61] M. Neek-Amal, A. Sadeghi, G.R. Berdiyorov, F.M. Peeters, Appl. Phys. Lett. 103 (2013) 261904.

[62] W. Hu, T. Wang, J. Yang, J. Mater. Chem. C 3 (2015) 4756.

[63] J.E. Padilha, A. Fazzio, A.J.R. da Silva, Phys. Rev. Lett. 103 (2015) 066803.

[64] A. Avsar, I.J. Vera-Marun, J.Y. Tan, K. Watanabe, T. Taniguchi, A.H.C. Neto, B. Özyilmaz, ACS Nano 9 (2015) 4138

[65] Q.J. Xiang, J.G. Yu, M. Jaroniec, J. Phys. Chem. C 115 (2011) 7355

[66] X.-H. Li, J.-S. Chen, X. Wang, J. Sun, M. Antonietti, J. Am. Chem. Soc. 133 (2011) 8074.

[67] A. Du, S. Sanvito, Z. Li, D. Wang, Y. Jiao, T. Liao, Q. Sun, Y.H. Ng, Z. Zhu, R. Amal, S.C. Smith, J. Am. Chem. Soc. 134 (2012) 4393.

[68] W. Hu, Z. Li, J. Yang, J. Chem. Phys. 138 (2013) 124706 .

[69] W. Geng, X. Zhao, H. Liu, X. Yao, J. Phys. Chem. C 117 (2013) 10536.

[70] X. Guo, Y.G. Zhou, J. Appl. Phys. 113 (2013) 054307.

[71] Y. Ma, Y. Dai, M. Guo, C. Niu, B. Huang, Nanoscale 3 (2011) 3883.

[72] X. D. Li, S. Yu, S.Q. Wu, Y.H. Wen, S. Zhou, Z.Z. Zhu, J. Phys. Chem. C 117 (2013) 15347.

[73] L. Britnell, R.M. Ribeiro, A. Eckmann, R. Jalil, B.D. Belle, A. Mishchenko, Y.-J. Kim, R.V. Gorbachev, T. Georgiou, S.V. Morozov, A.N. Grigorenko, A.K. Geim, C. Casiraghi, A.H.C. Neto, K.S. Novoselov, Science 340 (2013) 1311.
[74] N. Myoung, K. Seo, S.J. Lee, G. Ihm, ACS Nano 7 (2013) 7021

[75] W. Hu, T. Wang, J. Yang, arXiv:1411.0357 [condmat.mtrl-sci] (2014).

[76] J.N. Coleman, M. Lotya, A. O’Neill, S.D. Bergin, P.J. King, U. Khan, K. Young, A. Gaucher, S. De, R.J. Smith, I.V. Shvets, S.K. Arora, G. Stanton, H.-Y. Kim, K. Lee, G.T. Kim, G.S. Duesberg, T. Hallam, J.J. Boland, J.J. Wang, J.F. Donegan, J.C. Grunlan, G. Moriarty, A. Shmeliov, R.J. Nicholls, J.M. Perkins, E.M. Grieveson, K. Theuwissen, D.W. McComb, P.D. Nellist, V. Nicolosi, Science 331 (2011) 568.

[77] Y. Ma, Y Dai, W. Wei, C. Niu, L. Yu, B. Huang, J. Phys. Chem. C 115 (2011) 20237

[78] H. Tang, K. Dou, C.-C. Kaun, Q. Kuang, S, Yang, J. Mater. Chem. A 2 (2014) 360.

[79] Z. Ni, Q. Liu, K. Tang, J. Zheng, J. Zhou, R. Qin, Z. Gao, D. Yu, J. Lu, Nano Lett. 12 (2012) 113.

[80] H. Liu, J. Gao, J. Zhao, J. Phys. Chem. C 117 (2013) 10353.

[81] Z. Guo, S. Furuya, J. Iwata, A. Oshiyama, Phys. Rev. B 87 (2013) 235435.

[82] X.D. Li, S.Q. Wu, S. Zhou, Z.Z. Zhu, Nanoscale Res. Lett. 9 (2014) 110.

[83] L. Li, M. Zhao, J. Phys. Chem. C 118 (2014) 19129.

[84] N. Gao, J.C. Li, Q. Jiang, Phys. Chem. Chem. Phys. 16 (2014) 11673.

[85] N. Lu, H. Guo, L. Li, J. Dai, L. Wang, W.-N. Mei, X. Wu, X.C. Zeng, Nanoscale 6 (2014) 2879.

[86] N. Lu, H. Guo, L. Wang, X. Wu, X.C. Zeng, Nanoscale 6 (2014) 4566.

[87] J. Wang, Z. Guan, J. Huang, Q. Li, J. Yang, J. Mater. Chem. A 2 (2014) 7960.

[88] G. Kresse, J. Hafner, Phys. Rev. B 47 (1993) 558

[89] G. Makov, M.C. Payne, Phys. Rev. B 51 (1995) 4014.

[90] J.P. Perdew, K. Burke, M. Ernzerhof, Phys. Rev. Lett. 77 (1996) 3865.

[91] S. Grimme, J. Comput. Chem. 25 (2004) 1463.

[92] S. Grimme, J. Comput. Chem. 27 (2006) 1787.

[93] S. Grimme, S. Ehrlich, L. Goerigk, J. Comput. Chem. 32 (2011) 1456.

[94] M. Dion, H. Rydberg, E. Schröder, D.C. Langreth, B.I Lundqvist, Phys. Rev. Lett. 92 (2004) 246401.

[95] G. Román-Pérez, J.M. Soler, Phys. Rev. Lett. 103 (2009) 096102.

[96] K. Lee, E.D. Murray, L. Kong, B.I. Lundqvist, D.C. Langreth, Phys. Rev. B 82 (2010) 081101.

[97] R. Zacharia, H. Ulbricht, T. Hertel, Phys. Rev. B 69 (2004) 155406.

[98] R.E. Mapasha, A.M. Ukpong, N. Chetty, Phys. Rev. B 85 (2012) 205402.

[99] S. Grimme, C. Muck-Lichtenfeld,J. Antony, J. Phys. Chem. C 111 (2007) 11199.

[100] J. Antony, S. Grimme, Phys. Chem. Chem. Phys. 10 (2008) 2722.

[101] N. Kharche, S.K. Nayak, Nano Lett. 11 (2011) 5274

[102] J. Sławińska, P. Dabrowski, I. Zasada, Phys. Rev. B 83 (2011) 245429.

[103] R. Kagimura, M.S.C. Mazzoni, H. Chacham, Phys. Rev. B 85 (2012) 125415.

[104] Y. Ma, Y. Dai, M. Guo, B. Huang, Phys. Rev. B 85 (2012) 235448.

[105] L. Chen, L. Wang, Z. Shuai, D. Beljonne, J. Phys. Chem. Lett. 4 (2013) 2158. 
[106] W. Hu, X. Wu, Z. Li, J. Yang, Nanoscale 5 (2013) 9062.

[107] W. Hu, X. Wu, Z. Li, J. Yang, Phys. Chem. Chem. Phys. 15 (2013) 5753.

[108] W. Hu, N. Xia, X. Wu, Z. Li, J. Yang, Phys. Chem. Chem. Phys. 16 (2014) 6957

[109] W. Xia, W. Hu, Z. Li, J. Yang, Phys. Chem. Chem. Phys. 16 (2014) 22495.

[110] M. Gajdoš, K. Hummer, G. Kresse, Phys. Rev. B 73
(2006) 045112.

[111] J. Bardeen, Phys. Rev. 71 (1947) 717.

[112] G. Giovannetti, P.A. Khomyakov, G. Brocks, V.M. Karpan, J. van den Brink, P.J. Kelly, Phys. Rev. Lett. 101 (2008) 026803.

[113] W. Chen, E.J.G. Santos, W. Zhu, E. Kaxiras, Z. Zhang, Nano Lett. 13 (2013) 509. 
Silicene
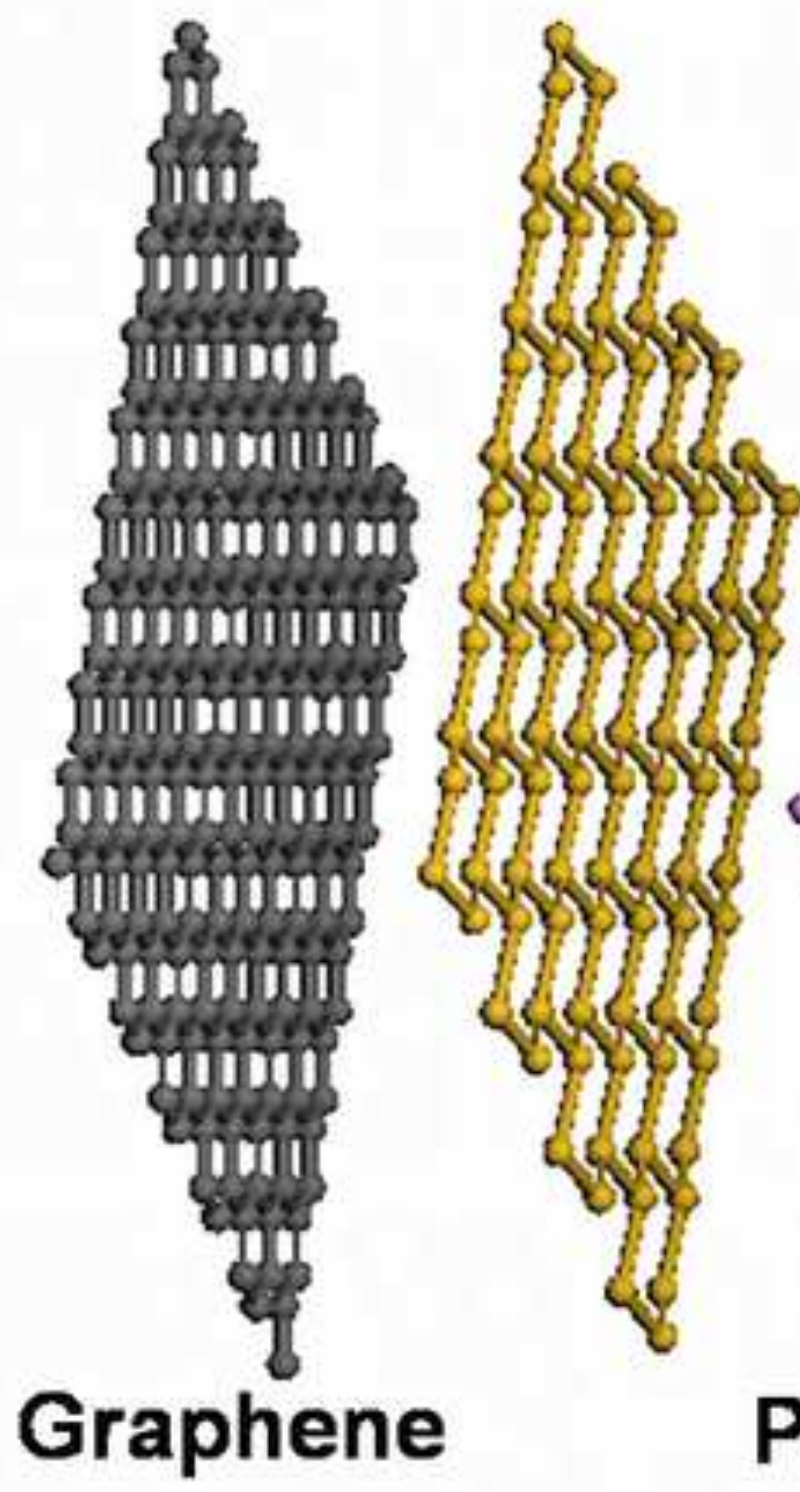

Phosphorene
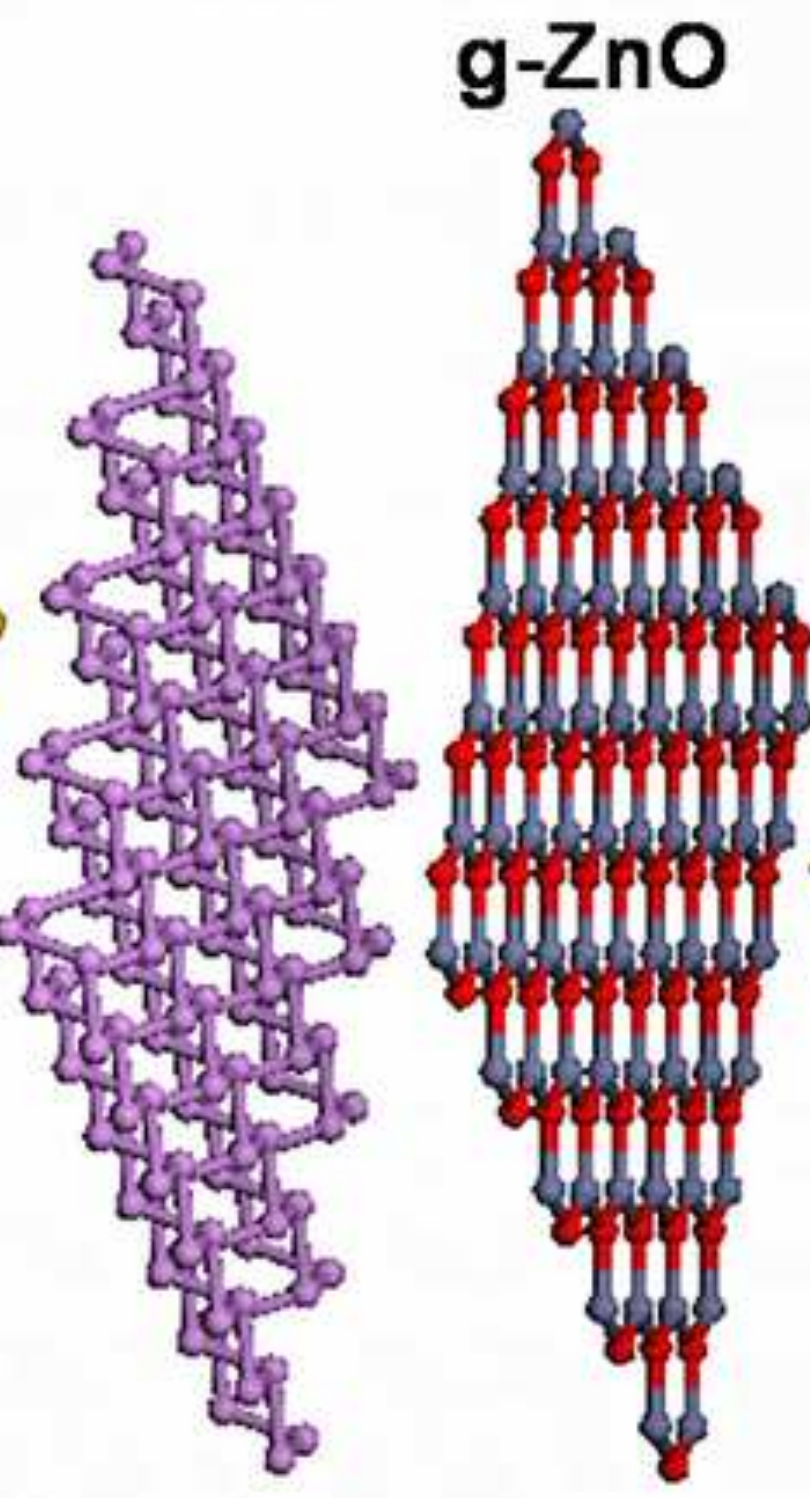

con 3 is

6 की

(c)

cind?

Ronso

2h

20

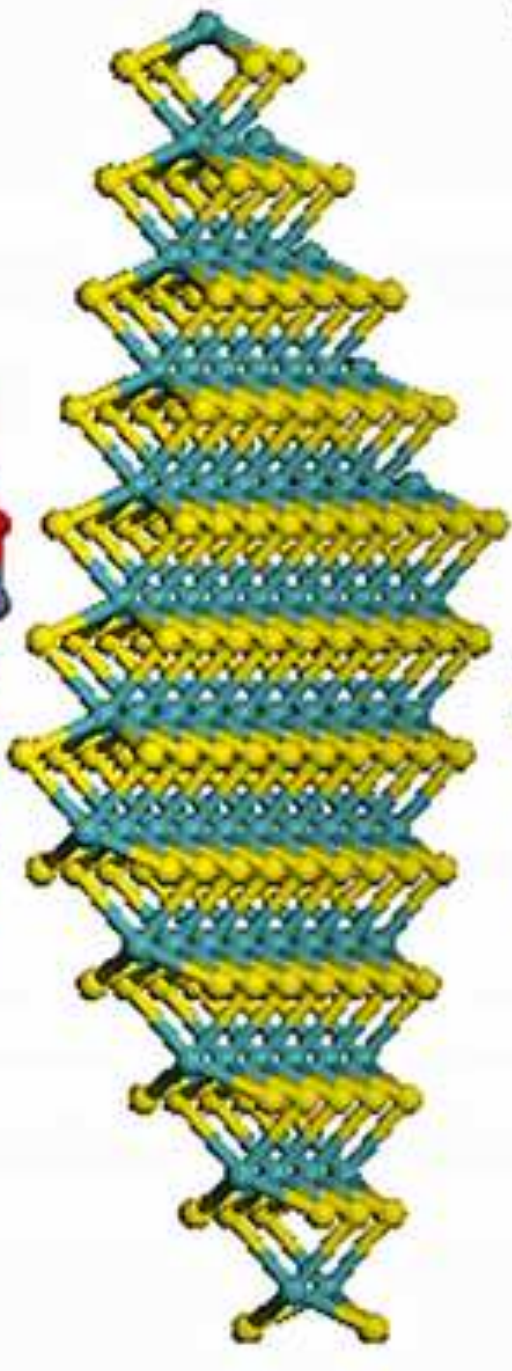

g- $\mathrm{C}_{3} \mathrm{~N}_{4}$

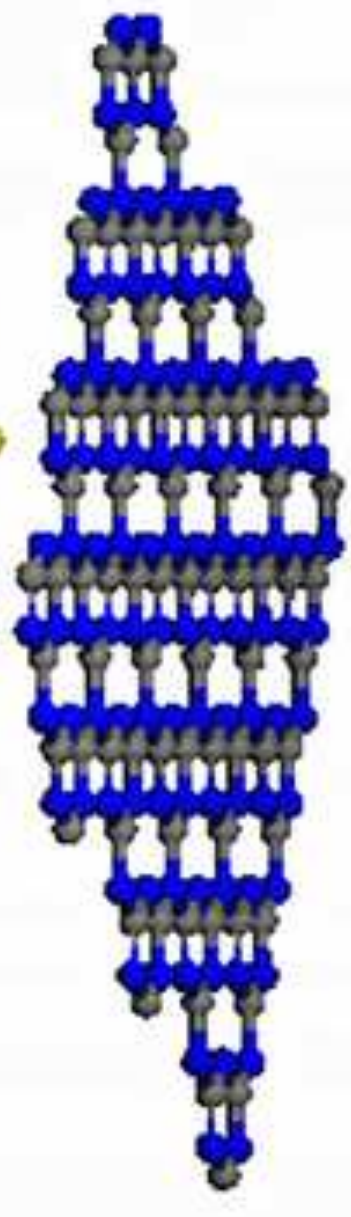

$\mathrm{MoS}_{2}$ 